\title{
STRENGTH OF CERTAIN FUZZY GRAPHS
}

\author{
K.P. Chithra ${ }^{1}$, Raji Pilakkat ${ }^{2}$ \\ ${ }^{1}$ Department of Mathematics \\ University of Calicut \\ INDIA
}

\begin{abstract}
In this paper we find the strength of a strong fuzzy wheel graph, strong fuzzy complete bipartite graph, strong fuzzy butterfly graph, strong fuzzy bull graph and also determined the strength of a properly linked fuzzy graph with complete fuzzy graphs as its parts.
\end{abstract}

AMS Subject Classification: $05 \mathrm{C} 72$

Key Words: strength of fuzzy graphs, fuzzy wheel graph, fuzzy hub, fuzzy butterfly graph, n-linked fuzzy graphs, fuzzy bull graph

\section{Introduction}

In 1965, Lofti A. Zadeh [7] introduced the notion of a fuzzy subset. Azriel Rosenfeld [5] defined fuzzy graph based on the definitions of fuzzy sets and fuzzy relations and developed the theory of fuzzy graphs in 1975. John N. Mordeson and Premchand S. Nair [3] introduced different types of operations on fuzzy graphs. Sheeba M.B.[6] introduced the concept of strength of fuzzy graphs. She determined the strength of fuzzy graphs in two different ways. One by introducing weight matrix of a fuzzy graph and the other by introducing the concept of extra strong path between its vertices. In this paper we use the concept of extra strong path to find the strength of various fuzzy graphs.

Throughout this paper only undirected fuzzy graphs are considered.

Received: October 19, 2015

Published: February 27, 2016

${ }^{\S}$ Correspondence author
(C) 2016 Academic Publications, Ltd.

url: www.acadpubl.eu 


\subsection{Preliminaries}

A fuzzy graph $G=(V, \mu, \sigma)[3]$ is a nonempty set $V$ together with a pair of functions $\mu: V \longrightarrow[0,1]$ and $\sigma: V \times V \longrightarrow[0,1]$ such that for all $u, v \in V$, $\sigma(u, v)=\sigma(u v) \leq \mu(u) \wedge \mu(v)$. We call $\mu$ the fuzzy vertex set of $G$ and $\sigma$ the fuzzy edge set of $G$. Here after we denote the fuzzy graph $G(\mu, \sigma)$ simply by $G$ and the underlying crisp graph of $G$ by $G^{*}(V, E)$ with $V$ as vertex set and $E=\{(u, v) \in V \times V: \sigma(u, v)>0\}$ as the edge set or simply by $G^{*}$. If for $(u, v) \in E$ we say that $u$ and $v$ are adjacent in $G^{*}$. In that case we also say that $u$ and $v$ are adjacent in $G$. A fuzzy graph $G$ is complete [3] if $\sigma(u v)=\mu(u) \wedge \mu(v)$ for all $u, v \in V$. A fuzzy graph $G$ is a strong fuzzy graph [3] if $\sigma(u v)=\mu(u) \wedge \mu(v), \forall u v \in E$. The strength of a strong fuzzy complete graph is one [6]. Let $G_{1}\left(V_{1}, \mu_{1}, \sigma_{1}\right)$ and $G_{2}\left(V_{2}, \mu_{2}, \sigma_{2}\right)$ be two fuzzy graphs with the underlying crisp graphs $G_{1}\left(V_{1}, X_{1}\right)$ and $G_{2}\left(V_{2}, X_{2}\right)$ respectively. If $V_{1} \cap V_{2}=\phi$ then their join [3] is the fuzzy graph $G=G_{1} \vee G_{2}\left(V_{1} \cup V_{2}, \mu_{1} \vee \mu_{2}, \sigma_{1} \vee \sigma_{2}\right)$ with the underlying crisp graph $G^{*}=\left(V_{1} \cup V_{2}, X_{1} \cup X_{2} \cup X^{\prime}\right)$ where $X^{\prime}$ is the set of all edges joining the vertices of $V_{1}$ and $V_{2}$ and

$$
\begin{gathered}
\left(\mu_{1} \vee \mu_{2}\right)(u)= \begin{cases}\mu_{1}(u) & \text { if } u \in V_{1} \backslash V_{2}, \\
\mu_{2}(u) & \text { if } u \in V_{2} \backslash V_{1}\end{cases} \\
\left(\sigma_{1} \vee \sigma_{2}\right)(u v)= \begin{cases}\sigma_{1}(u v) & \text { if } u v \in X_{1} \backslash X_{2}, \\
\sigma_{2}(u v) & \text { if } u v \in X_{2} \backslash X_{1}, \\
\mu_{1}(u) \wedge \mu_{2}(v) & \text { if } u \in X_{1} \text { and } v \in X_{2} .\end{cases}
\end{gathered}
$$

A strong fuzzy complete bipartite graph is a strong fuzzy graph with its underlying crisp graph is a complete bipartite graph [4]. A path $P$ of length $n-1$ in a fuzzy graph $G[3]$ is a sequence of distinct vertices $v_{1}, v_{2}, v_{3}, \ldots, v_{n}$, such that $\sigma\left(v_{i}, v_{i+1}\right)>0, i=1,2,3, \ldots, n-1$. We call $P$ a fuzzy cycle if $v_{1}=v_{n}$ and $n \geq 3$ and there does not exist a unique edge such that $\sigma(x y)=$ $\wedge\{\sigma(u v) /(u v)>0\}$. The strength of a path is defined as the weight of the weakest edge of the path [3]. A path $P$ is said to connect the vertices $u$ and $v$ of $G$ strongly if its strength is maximum among all paths between $u$ and $v$. Such paths are called strong paths [7]. Any strong path between two distinct vertices $u$ and $v$ in $G$ with minimum length is called an extra strong path between them [6]. There may exists more than one extra strong paths between two vertices in a fuzzy graph $G$. But, by the definition of an extra strong path each such path between two vertices has the same length. The maximum length of extra strong paths between every pair of distinct vertices in $G$ is called the strength of the graph $G$ [6]. For a fuzzy graph $G$, if $G^{*}$ is the path $P=v_{1} v_{2} \ldots v_{n}$ on $n$ vertices then the strength of the graph $G$ is its length $(n-1)[6]$. 
Here after for a fuzzy graph $G$ we use $\mathscr{S}(G)$ to denote its strength. The following theorems determine the strength of a fuzzy cycle.

Theorem 1. [6] In a fuzzy cycle $G$ of length $n$, suppose there are $l$ weakest edges where $l \leq\left[\frac{n+1}{2}\right]$. If these weakest edges altogether form a subpath then $\mathscr{S}(G)$ is $n-l$.

Theorem 2. [6] Let $G$ be a fuzzy cycle with crisp graph $G^{*}$ a cycle of length $n$, having $l$ weakest edges which altogether form a subpath. If $l>\left[\frac{n+1}{2}\right]$, then $\mathscr{S}(G)$ is $\left[\frac{n}{2}\right]$.

Theorem 3. [6] Let $G$ be a fuzzy cycle with crisp graph $G^{*}$ a cycle of length $n$, having $l$ weakest edges which do not altogether form a subpath. If $l>\left[\frac{n}{2}\right]-1$ then the strength of the graph is $\left[\frac{n}{2}\right]$ and if $l=\left[\frac{n}{2}\right]-1$ then $\mathscr{S}(G)$ is $\left[\frac{n+1}{2}\right]$.

Theorem 4. [6] In a fuzzy cycle of length $n$ suppose there are $l<\left[\frac{n}{2}\right]-$ 1 weakest edges which do not altogether form a subpath. Let $s$ denote the maximum length of a subpath which does not contain any weakest edge. If $s \leq\left[\frac{n}{2}\right]$ then the strength of the graph is $\left[\frac{n}{2}\right]$ and if $s>\left[\frac{n}{2}\right]$ then the strength of the graph is $s$.

\section{Main Results}

Lemma 5. Let $G$ be a strong fuzzy graph. If $u$ and $v$ are two adjacent vertices of $G$ then the length of the extra strong path joining $u$ and $v$ is one.

Proof. Suppose that $u$ and $v$ are adjacent in $G$. Since $G$ is a strong fuzzy graph, the edge $u v$, which is a path $u-v$ joining $u$ and $v$, has strength $\mu(u) \wedge$ $\mu(v)$. All the other paths joining $u$ and $v$ have strength less than or equal to $\mu(u) \wedge \mu(v)$. Hence the edge $u v$ is the unique extra strong path joining $u$ and $v$. Hence the result.

Remark 1. Let $G$ be a fuzzy graph. Suppose $u$ and $v$ are two adjacent vertices of $G$. Then the edge $u v$ is the only extra strong path joining $u$ and $v$. So for the computation of strength of a fuzzy graph we need only to consider its distinct non-adjacent vertices.

We introduce the following definitions to get a better understanding of the proof of the theorems that we are going to prove.

Definition 6. A fuzzy graph with $K_{1}[2]$ as its underlying graph is called a fuzzy vertex and it is also denoted by $K_{1}$. 
Definition 7. A fuzzy wheel graph $W_{n}$ is the join of the fuzzy cycle $C_{n-1}$ and the fuzzy vertex $K_{1}$.

Definition 8. A vertex $h$ of the wheel graph $W_{n}$ is said to be a fuzzy hub if it is adjacent to all the other vertices of $W_{n}$.

Definition 9. A strong fuzzy wheel graph is a fuzzy wheel graph which is also a strong fuzzy graph.

Theorem 10. For $n \geq 4$, let $W_{n}=C_{n-1} \vee K_{1}$ be a strong fuzzy wheel graph with the fuzzy hub $h$ and $u_{1} u_{2} \ldots u_{n-1} u_{1}$ the fuzzy cycle $C_{n-1}$. If $\mu(h)<$ $\mu\left(u_{i}\right), \forall i=1,2, \ldots, n-1$ then the strength of $W_{n}$ is the strength of $C_{n-1}$.

Proof. Since $h$ is adjacent to all the $u_{i}$ s, $i=1,2, \ldots, n-1$, length of all $h-u_{i}$ paths are one by Lemma 5 .

Choose two distinct non-adjacent vertices $u_{i}$ and $u_{j}$ of $C_{n-1}$. Since $\mu(h)<$ $\mu\left(u_{i}\right), i=1,2, \ldots, n-1$, all paths joining $u_{i}$ and $u_{j}$, through $h$ have strength $\mu(h)$, which is less than the strength of any path joining $u_{i}$ and $u_{j}$ in $C_{n-1}$. Therefore the length of extra strong paths joining $u_{i}$ and $u_{j}$ in $W_{n}$ and those in $C_{n-1}$ are one and the same. Hence the result.

Theorem 11. Let $W_{n}$ be as in Theorem 10. If $\mu(h) \geq \mu\left(u_{i}\right), \forall i=$ $1,2, \ldots, n-1$ then the strength of $W_{n}$ is one when $n=4$ and two when $n>4$.

Proof. When $n=4, W_{n}$ is a complete fuzzy graph. Therefore the strength of $W_{n}$ is one [6].

Now suppose that $n>4$. Let $u$ and $v$ be any two distinct non-adjacent vertices of $W_{n}$. Therefore both belong to $V\left(C_{n-1}\right)$ and the length of any extra strong path joining $u$ and $v$ is greater than or equal to 2. Without loss of generality assume that $\mu(u) \leq \mu(v)$. So all the paths joining $u$ and $v$ have strength less than or equal to $\mu(u)$. Therefore we can conclude that $u h v$ is an extra strong path between $u$ and $v$. So strength of $W_{n}$ is 2 .

The only remaining case is that some vertices of $C_{n-1}$ have weight greater than $\mu(h)$ and some have weight less than or equal to $\mu(h)$. In this case we have,

Theorem 12. Let $W_{n}$ be as in Theorem 10. Suppose that $\mu(h) \leq \mu\left(u_{i}\right)$ for some but not all the vertices $u_{i}, i=1,2, \ldots, n-1$. Let $P$ be one of the maximal paths of $C_{n-1}$ with the property that each edge of which has strength greater than $\mu(h)$. Let $l$ be the length of $P$. Then 


$$
\mathscr{S}\left(W_{n}\right)= \begin{cases}l & \text { if } l \geq 2 \\ 2 & \text { if } l=1 .\end{cases}
$$

Proof. Let $u, v$ be any two distinct non-adjacent vertices of $W_{n}$. Then $u$ and $v$ are vertices of $C_{n-1}$. Let $P_{1}$ and $P_{2}$ be two paths in $C_{n-1}$ having $u$ and $v$ as the end vertices.

Suppose that $l=1$. Then both $P_{1}$ and $P_{2}$ have strength less than or equal to $\mu(h)$. Therefore $u h v$ is an extra strong path in $W_{n}$. Hence in this case $\mathscr{S}\left(W_{n}\right)=2$.

Now suppose $l \geq 2$. If both the paths $P_{1}$ and $P_{2}$ have strength less than or equal to $\mu(h)$ then $u h v$ is an extra strong path joining $u$ and $v$ and which is of length 2. If exactly one of the paths $P_{1}$ and $P_{2}$ say $P_{1}$ has strength greater than $\mu(h)$, then the extra strong path joining $u$ and $v$ in $W_{n}$ is the path $P_{1}$. Since each edge of which has strength greater than $\mu(h)$, the length of $P_{1} \leq$ length of $P=l$. In particular if $u$ and $v$ are the end vertices of $P$, then $P$ itself is an extra strong path joining $u$ and $v$. Hence the theorem.

Theorem 13. Strength of a strong fuzzy complete bipartite graph is two.

Proof. Let $G$ be a strong fuzzy complete graph with $K_{m n}$ as its underlying crisp graph. Let $U=\left\{u_{1}, u_{2}, \ldots, u_{m}\right\}$ and $V=\left\{v_{1}, v_{2}, \ldots, v_{n}\right\}$ be the bipartite sets. Also let $u$ and $v$ be any two distinct non-adjacent vertices of $G$. If $u$ and $v \in U$, then all the $u-v$ paths in $G$ pass through atleast one vertex in $V$. Let $w$ be a vertex in $V$ with $\mu(w) \geq \mu\left(v_{i}\right), \forall v_{i} \in V$. Then strength of any $u-v$ path is less than or equal to that of the path $u w v$ in $G$. Therefore $u w v$ is one of the extra strong paths joining $u$ and $v$ and which is of length 2. Similar is the case when both $u, v \in V$. Hence the theorem.

Definition 14. A finite sequence of fuzzy graphs $G_{1}, G_{2}, \ldots, G_{m}$ with the property that $V\left(G_{i}\right) \cap V\left(G_{j}\right)$ is nonempty only if $|j-i| \leq 1,1 \leq i, j \leq m$ is called a properly linked sequence or simply properly linked. It is $n$ - linked if the crisp graph induced by $V\left(G_{i}\right) \cap V\left(G_{j}\right)$ is $K_{n}$, a complete graph on $n$ vertices, if $|j-i|=1,1 \leq i, j \leq m$.

Definition 15. A fuzzy graph $G$ is said to be properly linked ( $n$-linked) if there exists a finite sequence of properly linked partial fuzzy subgraphs $G_{1}, G_{2}, \ldots, G_{m}$, where $m>1$, such that $G=G_{1} \cup G_{2} \cup \ldots \cup G_{m}$. In this case we say that $G_{1}, G_{2}, \ldots, G_{m}$ are parts of $G$.

Notation 16. If a fuzzy graph $G$ is a union of a sequence of $m, n$-linked fuzzy graphs $G_{1}, G_{2}, \ldots, G_{m}$, then for any $n$ we write $G=G_{1}<G_{2}<\ldots<G_{m}$. 
Lemma 17. Let $G=G_{1}<G_{2}<\ldots<G_{m}$ be a 1-linked fuzzy graph with $G_{1}, G_{2}, \ldots, G_{m}$ (where $m>1$ ) as its parts. For, $i=1,2, \ldots, n-1$, let $V\left(G_{i}\right) \cap V\left(G_{i+1}\right)=\left\{v_{i}\right\}$. Let $u, v$ be any two distinct vertices of $G$. For $k<m$, if $u \in V\left(G_{1}\right) \backslash\left\{v_{1}\right\}$ and $v \in V\left(G_{k+1}\right) \backslash\left\{v_{k}\right\}$ then the length of extra strong path joining $u$ and $v$ in $G$ is $k+1$.

Proof. This result is proved by induction on $k$. When $k=1, u \in V\left(G_{1}\right) \backslash$ $\left\{v_{1}\right\}$ and $v \in V\left(G_{2}\right) \backslash\left\{v_{1}\right\}$. Therefore all the $u-v$ paths pass through $v_{1}$. Since $G$ is complete, the extra strong path joining $u$ and $v_{1}$ and the same for $v_{1}$ and $v$ are respectively $u v_{1}$ and $v_{1} v$. Therefore the length of the extra strong path joining $u$ and $v$ is 2 .

Now, let us assume the result is true for every $k \leq n<m-1$. Let $u \in$ $V\left(G_{1}\right) \backslash\left\{v_{1}\right\}$ and $v \in V\left(G_{n+2}\right) \backslash\left\{v_{n+1}\right\}$. Note that every $u-v$ path must pass through the vertex $v_{n+1}$. By induction hypothesis the length of every extra strong path joining $u$ and $v_{n+1}$ is $n+1$. Since $v_{n+1}$ and $v$ lie in $G_{n+2}$, the only extra strong path joining $v_{n+1}$ and $v$ is the edge $v_{n+1} v$. Hence the length of the extra strong path joining $u$ and $v$ is $n+2$. In fact there is only one extra strong path joining $u$ and $v$. Hence the lemma holds by induction.

The following theorem is an immediate consequence of Lemma 17.

Theorem 18. Let $G$ be a 1-linked fuzzy graph with $m$, where $m>1$, complete fuzzy graphs as its parts. Then the strength of $G$ is $m$, the diameter of $G$.

Definition 19. A strong fuzzy butterfly graph is a strong fuzzy graph with its underlying crisp graph is a butterfly graph.

Corollary 20. The strength of a strong fuzzy butterfly graph is two.

Proof. A strong fuzzy butterfly graph is a properly linked fuzzy graph with two complete fuzzy triangles as its parts. So by Theorem 18 the strength of a fuzzy butterfly graph is 2 .

Definition 21. A strong fuzzy bull graph is a strong fuzzy graph with its underlying crisp graph is a bull graph.

Corollary 22. The strength of a strong fuzzy bull graph is 3 .

Proof. A strong fuzzy bull graph is 1-linked by a sequence of 3 complete fuzzy graphs $G_{1}, G_{2}$ and $G_{3}$, where $G_{1}$ and $G_{2}$ are fuzzy paths on two vertices and $G_{3}$ a strong fuzzy triangle graph. So by Theorem 18 the strength of a strong fuzzy bull graph is three. 
We generalise Theorem 18 as follows.

Theorem 23. Let $G$ be a properly linked fuzzy graph with the complete fuzzy graphs $G_{1}, G_{2}, \ldots, G_{m}$ as its parts. Suppose for $i=1,2, \ldots, m-1$, $<V\left(G_{i}\right) \cap V\left(G_{i+1}\right)>=K_{n_{i}}$, a complete graph on $n_{i}$ vertices. Then the strength of $G$ is $m$, the diameter of $G$.

Proof. Let $V\left(G_{i}\right) \cap V\left(G_{i+1}\right)=\left\{u_{i 1}, u_{i 2}, \ldots, u_{i n_{i}}\right\}$, for $i=1,2, \ldots, m-1$. Let $u$ and $v$ be any two distinct non-adjacent vertices of $G$.

We prove the theorem in two steps.

Step 1: In this step we prove that if $u \in V\left(G_{1}\right) \backslash\left\{u_{11}, u_{12}, \ldots, u_{1 n_{1}}\right\}$ and $v \in V\left(G_{k+1}\right) \backslash\left\{u_{k 1}, u_{k 2}, \ldots, u_{k n_{k}}\right\}$ then the length of the extra strong $u-v$ path is $k+1$, where $k \leq m-1$.

We prove this result by induction on $k$. Assume that $k=1$.

Then every $u-v$ path lies completely in $G_{1} \cup G_{2}$. When $m=2$ it is obvious. Otherwise, any $u-v$ path can be written as $P_{1}+P_{2}+P_{3}+P_{4}$ where $P_{1}$ is a path from $u$ to some vertex $u_{2 i}$ of $\left\{u_{21}, u_{22}, \ldots, u_{2 n_{2}}\right\}$ in $G_{1} \cup G_{2}, P_{2}$ is a path from $u_{2 i}$ to some vertex $w$ in $G_{3} \cup G_{4} \cup \ldots \cup G_{m}, P_{3}$ is a path from $w$ to some vertex $u_{2 j}$ of $\left\{u_{21}, u_{22}, \ldots, u_{2 n_{2}}\right\}$ in $G_{3} \cup G_{4} \cup \ldots \cup G_{m}$ and $P_{4}$ is a path from $u_{2 j}$ to $v$ in $G_{1} \cup G_{2}$. Then clearly the path $P_{1}+u_{2 i} u_{2 j}+P_{4}$ has strength greater than or equal to that of $P_{1}+P_{2}+P_{3}+P_{4}$. So all the extra strong paths joining $u$ and $v$ lie completely in $G_{1} \cup G_{2}$.

Since $G_{1}$ and $G_{2}$ are complete fuzzy graphs, both $u$ and $v$ are adjacent to all the vertices of $\left\{u_{11}, u_{12}, \ldots, u_{1 n_{1}}\right\}$. If $\mu\left(u_{1 k}\right)=\max \left\{u_{11}, u_{12}, \ldots, u_{1 n_{1}}\right\}$, then $u u_{1 k} v$ is an extra strong path joining $u$ and $v$ in $G_{1} \cup G_{2}$ and is of length 2 .

Assume that the result is true for $k \leq n \leq m-2$. To prove the result for $k=n+1$, let $u \in V\left(G_{1}\right) \backslash\left\{u_{11}, u_{12}, \ldots, u_{1 n_{1}}\right\}$ and $v \in V\left(G_{n+2}\right) \backslash$ $\left\{u_{n+11}, u_{n+12} \ldots, u_{n+1 n_{n+1}}\right\}$. Then as above we prove that every extra strong path joining $u$ and $v$ lies completely in $G_{1} \cup G_{2} \cup \ldots \cup G_{n+2}$. When $n=m-2$, it is obvious. For $n<m-2$, if the result is not true then there exist a $u-v$ path in $G$ which passes through a vertex of $V\left(G_{n+3}\right) \backslash\left\{u_{n+21}, u_{n+22}, \ldots, u_{n+2 n_{n+2}}\right\}$. Then it must pass through atleast one vertex of the set $\left\{u_{n+21}, u_{n+22}, \ldots, u_{n+2 n_{n+2}}\right\}$. Any such path can be written as $P_{1}+P_{2}+P_{3}+P_{4}$, where $P_{1}$ is a path from $u$ to some vertex $u_{n+2 i}$ of the set $\left\{u_{n+21}, u_{n+22}, \ldots, u_{n+2 n_{n+2}}\right\}$ in $G_{1} \cup G_{2} \cup \ldots \cup G_{n+2}$, $P_{2}$ is a path from $u_{n+2 i}$ to $z$ in $G_{n+3} \cup G_{n+4} \cup \ldots \cup G_{m}, P_{3}$ is a path from $z$ to some vertex $u_{n+2 j}$ of $\left\{u_{n+21}, u_{n+22}, \ldots, u_{n+2 n_{n+2}}\right\}$ in $G_{n+3} \cup G_{n+4} \cup \ldots \cup G_{m}$ and $P_{4}$ is a path from $u_{n+2 j}$ to $v$ in $G_{1} \cup G_{2} \cup \ldots \cup G_{n+2}$. Clearly the path $P_{1}++u_{n+2 i} u_{n+2 j}+P_{4}$ has strength greater than or equal to that of $P_{1}+P_{2}+P_{3}+P_{4}$ and any such path can be written as sum of two paths $P, Q$ where $P$ is an extra strong path from $u$ to $w \in\left\{u_{n+11}, u_{n+12}, \ldots, u_{n+1 n_{n+1}}\right\}$ in 

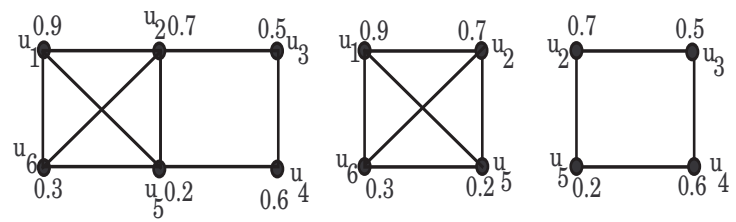

Figure 1: A 2-linked fuzzy graph $G$ (Left), its parts $G_{1}$ (Middle) and $G_{2}$ (Right).

$G_{1} \cup G_{2} \cup \ldots \cup G_{n+2}$, where $\mu(w)=\max \left\{\mu\left(u_{n+11}\right), \mu\left(u_{n+12}\right), \ldots, \mu\left(u_{n+1 n_{n+1}}\right)\right\}$ and $Q$ is the edge $w v$ of $G_{n+2}$, since $G_{n+2}$ is complete. Now by induction hypothesis the length of $P$ is $n+1$. Therefore the length of extra strong path joining $u$ and $v$ is $n+2$.

Step 2: Let $u$ and $v$ be any two vertices of $G$. Suppose $u$ and $v$ belong to the same part $G_{i}$ of $G$ then $u$ and $v$ are adjacent because $G_{i}$ is complete. Hence the edge $u v$ is the only extra strong $u-v$ path in $G$. Otherwise $u$ belongs to some $G_{i}$ and $v$ belongs to some $G_{j}$ of $G$, where $G_{i}$ and $G_{j}$ are two distinct parts of $G$. Without loss of generality assume that $i<j$. Then by Step 1 the length of extra strong path is $j-i+1 \leq m$. In particular when $i=1$ and $j=m$, the length of extra strong $u-v$ path is $m$. Hence $\mathscr{S}(G)=m$. Therefore the theorem follows.

Remark 2. Theorem 23 need not be true when atleast one part of a properly linked fuzzy graph fails to be a complete fuzzy graph. For example, the strong fuzzy graph $G$ in Figure 1 is a 2-linked fuzzy graph of strength 3 with parts $G_{1}$ and $G_{2}$, where $G_{1}$ is a complete fuzzy graph but $G_{2}$ is not a complete fuzzy graph.

Definition 24. A fuzzy diamond graph is a fuzzy graph with the underlying crisp graph is a diamond graph.

Corollary 25. The strength of a strong fuzzy diamond graph is 2 . 


\section{Conclusions}

In this paper we determined, the strength of a strong fuzzy wheel graph, strong fuzzy butterfly graph, strong fuzzy bull graph, strong fuzzy complete bipartite graph and strong fuzzy diamond graph. Also properly linked fuzzy graphs are defined and determined strength of certain such graphs.

\section{Acknowledgments}

The first author is grateful to University of Calicut for the fellowship under which this research was conducted.

\section{References}

[1] R. Balakrishnan, K. Ranganathan, Text Book of Graph Theory, Springer, (2008).

[2] J. A. Bondy, U. S. R. Murty, Graph Theory, Springer, (2008).

[3] N. John Mordeson, S. Premchand Nair, Fuzzy Graphs and Fuzzy Hypergraphs, PhysicaVerlag, Heidelberg, Second Edition, (2001).

[4] A. Nagoorgani, D. Rajalaxmi Subahashini, Fuzzy labeling tree, International Journal of Pure and Applied Mathematics, Volume 90 No. 2, (2014), 131-141.

[5] A. Rosenfeld, Fuzzy sets and their applications to cognitive and decision process, Academic press, New York (1975), 75-95.

[6] M. B. Sheeba, Strength of fuzzy graphs, Thesis for Ph.D Degree, University of Calicut, Calicut (2013).

[7] L. A. Zadeh, Fuzzy sets, Information and Control, California (1965), 338-353. 
